\title{
Integrating community-based health promotion programs and primary care: a mixed methods analysis of feasibility
}

Aaron L. Leppin ${ }^{1 *} \mathbb{D}$, Karen Schaepe ${ }^{2}$, Jason Egginton², Sara Dick', Megan Branda', Lori Christiansen³, Nicole M. Burow ${ }^{4}$, Charlene Gaw ${ }^{5}$ and Victor M. Montori ${ }^{1}$

\begin{abstract}
Background: Implementation of evidence-based programs (EBPs) for disease self-management and prevention is a policy priority. It is challenging to implement EBPs offered in community settings and to integrate them with healthcare. We sought to understand, categorize, and richly describe key challenges and opportunities related to integrating EBPs into routine primary care practice in the United States.

Methods: As part of a parent, participatory action research project, we conducted a mixed methods evaluation guided by the PRECEDE implementation planning model in an 11-county region of Southeast Minnesota. Our community-partnered research team interviewed and surveyed 15 and 190 primary care clinicians and 15 and 88 non-clinician stakeholders, respectively. We coded interviews according to pre-defined PRECEDE factors and by participant type and searched for emerging themes. We then categorized survey items-before looking at participant responses-according to their ability to generate further evidence supporting the PRECEDE factors and emerging themes. We statistically summarized data within and across responder groups. When consistent, we merged these with qualitative insight.

Results: The themes we found, "Two Systems, Two Worlds," "Not My Job," and "Seeing is Believing," highlighted the disparate nature of prescribed activities that different stakeholders do to contribute to health. For instance, primary care clinicians felt pressured to focus on activities of diagnosis and treatment and did not imagine ways in which EBPs could contribute to either. Quantitative analyses supported aspects of all three themes, highlighting clinicians' limited trust in community-placed activities, and the need for tailored education and system and policy-level changes to support their integration with primary care.
\end{abstract}

Conclusions: Primary care and community-based programs exist in disconnected worlds. Without urgent and intentional efforts to bridge well-care and sick-care, interventions that support people's efforts to be and stay well in their communities will remain outside of — if not at odds with — healthcare.

Keywords: CBPR, Community-based participatory research, Mixed methods, Implementation, Evidence-based programs, Chronic disease self-management program, Clinic-community linkages, Primary care, Chronic disease management

\footnotetext{
* Correspondence: Leppin.aaron@mayo.edu

${ }^{1}$ Knowledge and Evaluation Research Unit, Mayo Clinic, 200 First Street SW,

Rochester, MN 55905, USA

Full list of author information is available at the end of the article
} 


\section{Background}

Half of all American adults have at least one chronic condition; one in 4 live with the burdens of multiple chronic conditions [1]. When individuals with chronic illness are skilled at self-managing their health, they can persevere and thrive [2,3]. Unfortunately, many people lack the capacity, resources, and/or skills to live well with chronic disease [4-6]. Healthcare organizations desire to support patients in their journeys toward better health [7], but their usual isolation from the communities in which patients live limits their impact $[8,9]$. Interventions that occur in the places where people live their lives may better support the needs of patients with chronic illness. As such, multiple stakeholders are advocating for the implementation of evidence-based health promotion programs (EBPs) in the community [10-12].

EBPs are a collection of interventions that are developed and evaluated by researchers for the purpose of enhancing patient capacity to prevent and self-manage chronic health conditions. Typically, EBPs follow a scripted curriculum and are designed to be facilitated with high fidelity by trained lay leaders in community settings. Many have been shown in large trials to improve health outcomes and reduce costs [13-20].

The Chronic Disease Self-Management Program (CDSMP) is a key example [13]. The CDSMP is a group-based workshop delivered over 6 weekly sessions by two trained peer leaders and for a group of 8-15 participants with any chronic physical or mental health condition. Federally-sponsored evaluations of the program have proven its value $[14,21]$ and its widespread implementation is now a key priority of the United States Department of Health and Human Services (DHHS), the Agency for Healthcare Research and Quality (AHRQ), the Centers for Disease Control (CDC), and the National Council on Aging, among others [10, 12]. Arguably, half of the American adult population could benefit from participating in the CDSMP [21].

Because the CDSMP and other EBPs are offered outside of the traditional healthcare system and delivered by nontraditional healthcare workers, they present unique challenges for implementation [22, 23]. We sought to understand, categorize, and richly describe key challenges and opportunities related to integrating EBPs into routine primary care practice in the United States, using the CDSMP as a test case.

\section{Methods}

\section{Overview and setting}

The present study is a mixed methods, community engaged research evaluation informed by the Predisposing, Reinforcing, and Enabling Constructs in Environmental Diagnosis and Evaluation (PRECEDE) implementation planning model [24, 25]. It represents the preliminary research component of a parent, participatory action research [26] project focused on EBP implementation in an 11 county region of Southeast Minnesota. The parent project, which was designed to capitalize on the "partnership synergy" [27] of participatory implementation research [28], began when community stakeholders expressed a desire to implement the CDSMP in an effort to meet regional health needs. The community stakeholders were particularly interested in (1.) exploring barriers to clinical integration and (2.) developing strategies to promote referrals from healthcare to the community-based program. We used the PRECEDE planning model to guide these efforts because it has been used successfully in many similar projects $[25,29]$. Specifically, we oriented the model to assist-through a coded analysis of interviews and surveys-in identifying the predisposing, reinforcing, and enabling factors and the administrative and policy issues at multiple levels and across sectors that might be relevant to clinically integrating the CDSMP (see Table 1). We designed the study to include qualitative (interview) and quantitative (survey) components conducted in parallel, analyzed in series, and merged according to pre-defined methods (see Fig. 1). We collected both types of data at the same time and for the same exploratory purpose because this was most efficient. We waited, however, to design and conduct the quantitative analyses until after the qualitative analyses were complete. In this way, we hoped to ensure that the survey items corresponded to the appropriate PRECEDE constructs and that the quantitative data could be used to both strengthen and confirm the qualitative insight. We chose this approach-consistent with best practices for mixed methods research [30] - to gain rich and reliable understanding that would be of practical use in developing implementation strategies that would be both locally useful and broadly applicable [31].

\section{Measure development}

As part of the parent project, we convened a regional stakeholder meeting in the Spring of 2015. Attendees $(n=52)$ were educated about the PRECEDE model before reviewing, critiquing, and piloting interview guides and surveys for both primary care clinicians and other stakeholders loosely based on the model. Because we hypothesized that the beliefs and attitudes of the two groups (clinicians vs other stakeholders) would be different and because we wanted to explore this, many questions and items were the same on both versions. Topically, the measures covered issues of interest to the community stakeholders and related to knowledge and beliefs about both the CDSMP specifically and community resources in general and other factors-such as resources, norms, and policies-expected to affect their 
Table 1 The PRECEDE framework as conceptualized for this study

\begin{tabular}{|c|c|}
\hline PRECEDE Construct & Hypothetical Example \\
\hline $\begin{array}{l}\text { Predisposing Factors: the beliefs, } \\
\text { knowledge, and attitudes of } \\
\text { individuals that predispose them } \\
\text { to certain aligned behaviors }\end{array}$ & $\begin{array}{l}\text { Clinician knowledge of evidence in } \\
\text { support of the CDSMP }\end{array}$ \\
\hline $\begin{array}{l}\text { Reinforcing Factors: the community } \\
\text { norms, incentives, and } \\
\text { infrastructures that shape } \\
\text { and reinforce the }\end{array}$ & $\begin{array}{l}\text { Frequency with which clinicians' } \\
\text { peers and administrators discuss } \\
\text { the CDSMP }\end{array}$ \\
\hline
\end{tabular}

predisposing factors

Enabling Factors: the immediate availability of individual and community resources required to carry out aligned behaviors

Administrative Issues: the culture and priorities of a community or organization that determine, prescribe and facilitate changes to other factors

Policy Issues: the existing rules that require or prevent certain behaviors independent of motivations

implementation. To optimize response rates, the surveys were designed to be brief [32]. After synthesizing feedback, we posted revised versions of the guides and surveys for final stakeholder approval on a web-based project management tool (Basecamp). The Mayo Clinic Office of Community Engagement in Research approved the final versions of the interview guides and surveys and all study procedures. Copies of all measures are available in the Additional file 1: Online Supplement.

\section{Participants and recruitment}

For the qualitative component, we elected to interview 30 participants. We chose this sample size because we were familiar with the context and relevant stakeholders through prior collaborative work and we were confident it would be both feasible and sufficient to capture the breadth of insights. ALL recruited the participants, which consisted of 15 primary care clinicians ("clinicians") and 15 non-primary care clinician stakeholders ("stakeholders"). Clinicians were actively practicing physicians or nurse practitioners in the region. Stakeholders held a variety of titles (e.g. public health, patient, community agency, researcher, administrator) but were not actively practicing primary care; they were also known to have personal experience or special insight relevant to EBP implementation from either a community or healthcare perspective. (see Table 2) Participants in the quantitative component were also primary care clinicians practicing in the region (physicians, nurse practitioners, or physician assistants) and other stakeholders. Although most non-clinician stakeholders worked and resided within the region, some (e.g. state level stakeholders from the Department of Health) did not. Primary care clinicians for the quantitative analysis were recruited by contacting employing health systems $(n=5)$. A purposeful sample of other informed stakeholders was recruited through convenience sampling of currently engaged individuals (e.g. from the stakeholder meeting) and the contacts they recommended and provided. Six professional and 4 community researchers constituted the multidisciplinary research team. All community researchers were local professionals known to the PI from prior collaborative work. They had preexisting expertise and experience in EBP implementation as part of their professional roles and were recruited personally by the PI after being given a description of the anticipated requirements. Prior to study initiation they received training in the protection of human subjects and the conduct of qualitative interviews. Throughout the study the community researchers reported back to a larger group of stakeholders affiliated with the parent project who were not directly involved in the research conduct (this was so the other stakeholders could proceed with the "action" component of the parent project).

\section{Procedure}

Over a three-month period, an experienced qualitative researcher (JE) conducted semi-structured face-to-face and phone interviews of the 15 non-clinician stakeholders. During this same time, 4 community researchers

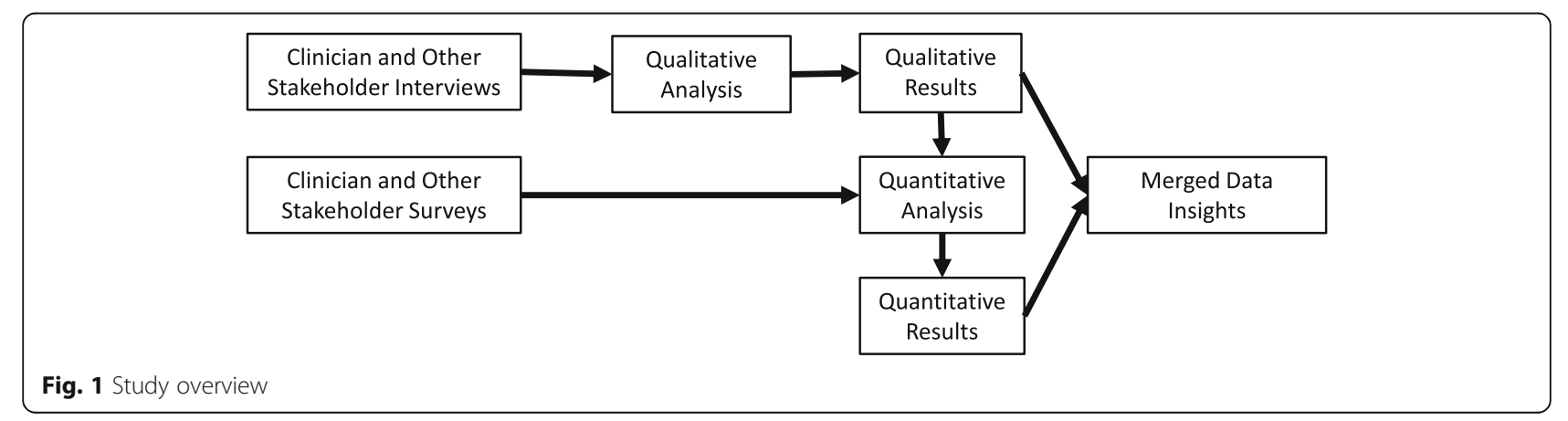


Table 2 Description of interview participants

\begin{tabular}{|c|c|c|c|}
\hline \multicolumn{2}{|c|}{ Primary care clinicians } & \multicolumn{2}{|c|}{$\begin{array}{l}\text { Other Stakeholders (Constituency } \\
\text { Represented) }\end{array}$} \\
\hline C101 & $\begin{array}{l}\text { Female, internal medicine } \\
\text { physician who oversees nurse } \\
\text { care coordinators for large system }\end{array}$ & 5001 & $\begin{array}{l}\text { Endocrinologist and researcher } \\
\text { with expertise in patient self- } \\
\text { management (healthcare system } \\
\text { structure informant) }\end{array}$ \\
\hline C102 & $\begin{array}{l}\text { Male, family medicine physician } \\
\text { who is on clinical practice } \\
\text { committee for large system }\end{array}$ & S002 & $\begin{array}{l}\text { Health services researcher and } \\
\text { patient familiar with CDSMP } \\
\text { (healthcare system structure } \\
\text { informant) }\end{array}$ \\
\hline C103 & $\begin{array}{l}\text { Female, internal medicine } \\
\text { physician at urban community } \\
\text { practice of large system }\end{array}$ & S003 & $\begin{array}{l}\text { Coordinator of EBPs for Area } \\
\text { Agency on Aging and CDSMP } \\
\text { leader (funded to implement } \\
\text { EBPs) }\end{array}$ \\
\hline C104 & $\begin{array}{l}\text { Female, internal medicine } \\
\text { physician at urban community } \\
\text { practice of large system }\end{array}$ & S004 & $\begin{array}{l}\text { Executive Director of Area } \\
\text { Agency on Aging (funded to } \\
\text { implement EBPs) }\end{array}$ \\
\hline C105 & $\begin{array}{l}\text { Male, internal medicine } \\
\text { physician leader in population } \\
\text { health of large system }\end{array}$ & S005 & $\begin{array}{l}\text { Health services researcher with } \\
\text { expertise in chronic care delivery } \\
\text { (healthcare system structure } \\
\text { informant) }\end{array}$ \\
\hline C106 & $\begin{array}{l}\text { Female, family medicine } \\
\text { physician leader in population } \\
\text { health for smaller system }\end{array}$ & S006 & $\begin{array}{l}\text { Director of community outreach } \\
\text { for small rural hospital affiliated } \\
\text { with large health system } \\
\text { (healthcare system structure } \\
\text { informant) }\end{array}$ \\
\hline C107 & $\begin{array}{l}\text { Female, family medicine } \\
\text { physician at urban community } \\
\text { practice of large system }\end{array}$ & S007 & $\begin{array}{l}\text { Public health employee tasked } \\
\text { with facilitating clinic-community } \\
\text { linkages in rural county (funded } \\
\text { to implement EBPs) }\end{array}$ \\
\hline C108 & $\begin{array}{l}\text { Male, family medicine physician } \\
\text { at urban community practice of } \\
\text { large system }\end{array}$ & S008 & $\begin{array}{l}\text { Employee of state Department of } \\
\text { Human Services with role in } \\
\text { CDSMP implementation (funded } \\
\text { to implement EBPs) }\end{array}$ \\
\hline C109 & $\begin{array}{l}\text { Male, internal medicine } \\
\text { physician at urban free clinic for } \\
\text { underserved }\end{array}$ & S009 & $\begin{array}{l}\text { Nurse care coordinator at rural } \\
\text { site in large health system } \\
\text { (healthcare system structure } \\
\text { informant) }\end{array}$ \\
\hline C110 & $\begin{array}{l}\text { Female, family medicine nurse } \\
\text { practitioner at rural clinic in } \\
\text { small health system }\end{array}$ & S010 & $\begin{array}{l}\text { Director of community outreach } \\
\text { for smaller health system and } \\
\text { CDSMP leader (healthcare system } \\
\text { structure informant) }\end{array}$ \\
\hline C111 & $\begin{array}{l}\text { Male, family medicine physician } \\
\text { assistant at rural clinic in small } \\
\text { health system }\end{array}$ & S011 & $\begin{array}{l}\text { Director of community } \\
\text { organization and CDSMP leader } \\
\text { (funded to implement EBPs) }\end{array}$ \\
\hline C112 & $\begin{array}{l}\text { Male, family medicine physician } \\
\text { at rural clinic for large health } \\
\text { system }\end{array}$ & S012 & $\begin{array}{l}\text { Nurse supervisor for nurse care } \\
\text { coordinators in large health } \\
\text { system (healthcare system } \\
\text { structure informant) }\end{array}$ \\
\hline C113 & $\begin{array}{l}\text { Female, family medicine } \\
\text { physician and medical director } \\
\text { of rural clinic for large system }\end{array}$ & S013 & $\begin{array}{l}\text { CDSMP leader at rural } \\
\text { rehabilitation center (funded to } \\
\text { implement EBPs) }\end{array}$ \\
\hline C114 & $\begin{array}{l}\text { Male, family medicine physician } \\
\text { at rural community practice of } \\
\text { large system }\end{array}$ & S014 & $\begin{array}{l}\text { Employee of state Department of } \\
\text { Health with role in EBP } \\
\text { implementation and CDSMP } \\
\text { leader (funded to implement } \\
\text { EBPs) }\end{array}$ \\
\hline C115 & $\begin{array}{l}\text { Male, family medicine physician } \\
\text { at urban community clinic }\end{array}$ & S015 & $\begin{array}{l}\text { Community leader working with } \\
\text { healthcare and focused on } \\
\text { health in urban development } \\
\text { (healthcare system structure } \\
\text { informant) }\end{array}$ \\
\hline
\end{tabular}

conducted similar interviews of the clinicians (each conducting 2-4 interviews). This approach was taken because we anticipated the clinician interviews to be less variable, more interesting, and less challenging technically for the community researchers. Interviews took approximately $30 \mathrm{~min}$ each; they were audiotaped and transcribed for analysis. Surveys of clinicians were distributed electronically or printed and distributed in person or by postal mail via complete or convenience sampling, depending on the preference of the participating healthcare systems. When possible, we sent printed surveys by postal mail to nonresponding clinicians who received electronic surveys. Other stakeholders received their surveys electronically via email; we sent electronic reminders to non-responders. We entered and stored all responses in a secure (RedCAP) [33] database for analysis.

\section{Analysis}

We conducted analyses in two phases (qual before quan) so as not to bias the qualitative interpretations. Using the PRECEDE framework, two team members (SD and JE) working independently and in duplicate, coded all the interviews. Specifically, interviewee comments were categorized into a priori codes of Predisposing, Enabling, and Reinforcing Factors, and Administrative and Policy Issues. After the initial coding, the team collectively reviewed transcripts to reconcile individual coding interpretations and confirm theoretical saturation. Narrative summaries were then distilled into brief and representative summary phrases and imported into one of two 5 (codes) by 15 (participants) frameworks for both clinicians and other stakeholders as appropriate. These frameworks were developed to provide a visual and actionable overview of the emerging information that the implementing stakeholders could understand and apply in the action phase of the parent project. To obtain a richer understanding of the key issues, a team of three experienced qualitative researchers (ALL, JE, $\mathrm{KS}$ ) reviewed the interview content and explored the ways in which the various PRECEDE factors interacted within and across participant groups. This process was used to develop additional, emergent themes and to gain more generalizable insights.

During the quantitative phase of the analysis, we categorized survey items-before looking at the data-by whether they were likely to generate insight that could triangulate with the qualitative PRECEDE codes. We calculated response rates as applicable and used standard descriptive statistics to characterize the clinician and non-clinician stakeholders and their responses. We tested for differences in proportions between groups with chi square tests of association. We followed a similar procedure for the quantitative assessment of the emergent themes with the exception that-for those themes that did not clearly correspond to a priori survey items-we constructed variables and analyses as feasible and appropriate (as determined by the authors). We 
conducted all quantitative analyses with SAS statistical software. Finally, we compared the collective insight from both the qualitative and quantitative analyses, judged for conceptual consistency, and took note of the ways in which methods and data sources confirmed, explained, and/or contradicted one another. Figure 1 shows an overview of the process we used to collect, analyze, and make sense of the data.

\section{Results}

Qualitative component

\section{Demographics}

We interviewed primary care clinicians from the three largest healthcare provider systems in Southeast Minnesota (a clinician from the fourth system declined to participate and we were unable to contact a clinician from the fifth system). Most were physicians that had some understanding of what the CDSMP was and the evidence supporting it. Only one, however, had successfully referred a patient to the program. The other interviewees were a diverse group of local and state level stakeholders. Eight were from the community and seven worked within a healthcare provider system in either a research or clinical capacity (Table 2).

\section{PRECEDE analysis}

Overall, we found relevant discussion mapping to all PRECEDE codes in both participant groups and examples of key barriers identified, organized by PRECEDE construct, are presented in Fig. 2. For a detailed table of representative quotes from clinician and stakeholder interview participants organized by PRECEDE construct, see the Additional file 1: Online Supplement. Here, we emphasize the overarching and emergent themes that arose from the deep exploration of the within and cross group interactions of these codes: namely, "Two Systems, Two Worlds," "Not My Job," and "Seeing Is Believing."

\section{Emergent themes analysis}

The theme of Two Systems, Two Worlds highlighted the importance of key policy and administrative issues in determining the different priorities of healthcare and the community. Superficially, it was supported by the fact that non-clinician stakeholders within the healthcare system seemed to offer insights more similar to those of clinicians than to those of their community-based counterparts in regards to both tone and topic. This discordance appeared to be rooted in key policy and administrative issues that determined that the priorities of-and associated resources and types of knowledge available to-participants within and outside of the healthcare system be different. Key community stakeholders, for example, were familiar with the CDSMP and other EBPs because of Federal and state-level policies and initiatives that funded and/or encouraged their particular implementation of them. This was especially true at the local Area Agency on Aging, where EBP implementation was the entire focus of one employee's job. She even described the policy-enacted support she had at her disposal to do this work, claiming it was too directive and limited:

"So a portion of the [EBP] workshops that go on are funded under Older Americans Act Title IIID funding, which is, that funding has to be used specifically for evidence-based health promotion programs but they also have to be geared toward seniors. So that's a limitation of that. And then another portion of workshops going on, the [providing] organizations are just taking it on as part of what they do as an organization."

This approach of engaging organizations to take programs on as "what they do" was a key strategy for scaling EBP implementation in the setting of fixed resources. And, as it turned out, the most accessible and willing partners were typically community-based non-profits. The activities

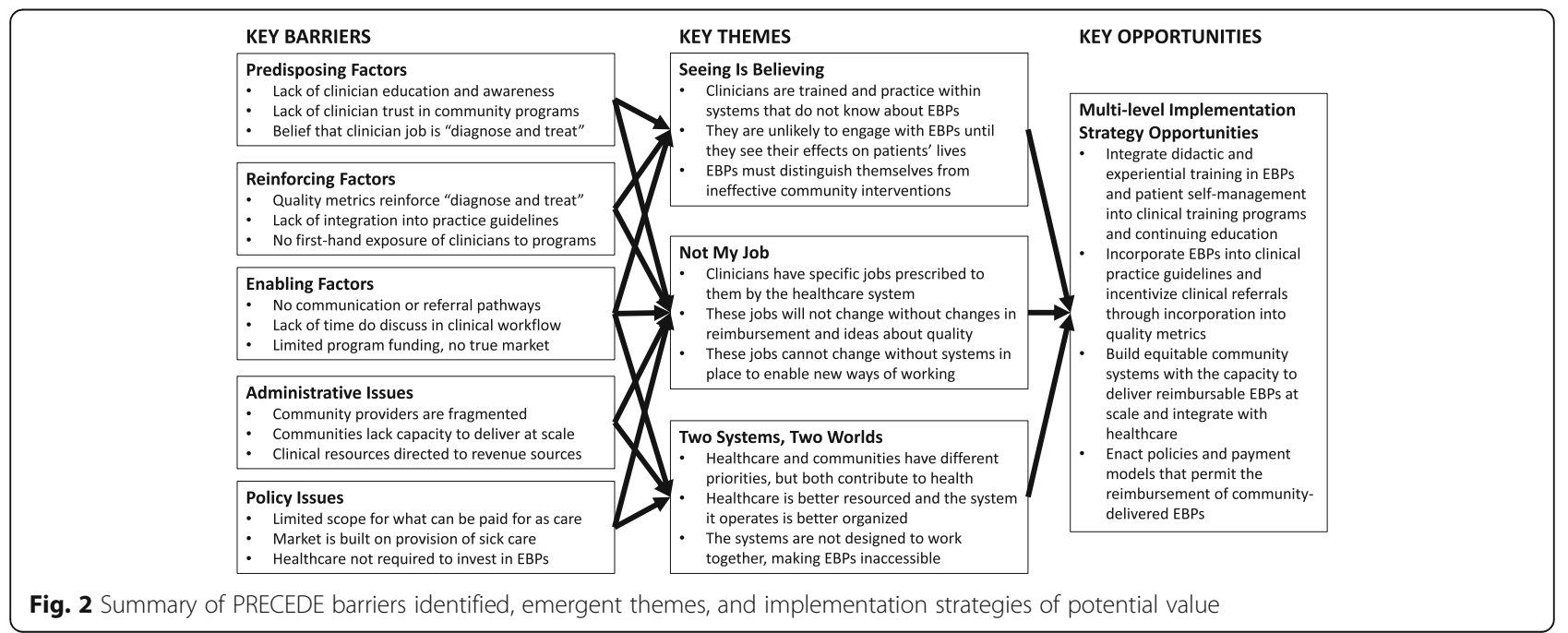


these organizations undertook together to coordinate the training of leaders and licensing and delivery of programs seemed to contribute to the development of a "community system" of trust, familiarity, and purpose, and to the development of a common way of working. Perhaps consequently-and reflective of and reinforced by past efforts-the community stakeholders viewed the CDSMP as something they mostly did (or were trying to do) independent of the healthcare system. Ironically, this was not their desire, and many interviewees highlighted the need for clinical referrals to the programs for success and sustainability. Indeed, clinical integration had apparently always been the hope and expectation of EBP implementation; but, many were giving up on that ambition. As one reflected:

"Nationally, when all of these evidence-based programs were funded by the Older Americans Act, the thought was that we were gonna educate doctors and they were gonna be telling patients about these programs and classes, but that's not realistic. You know their time, ah, is so prescribed in many of the clinics across the country that that's just not a feasible approach."

The clinicians we interviewed uniformly agreed that their time was heavily prescribed, and that making referrals to the CDSMP and other EBPs was not part of what they were being encouraged to do. In that respect, it seemed that the policy and administrative issues of the healthcare system were not well matched to those of the community. As one clinician said:

"Part of the issue [preventing clinicians from making referrals] may be you know there, there are so many things coming at, at us in healthcare these days. Including expectations and requirements about different ways to care for patients. About things we have to meet for metrics and measures."

Although they were often a source of burden and frustration, clinicians felt most responsible for these "metrics and measures"-typically based on clinical practice guidelines for disease screening and treatment protocols. The reason these measures were prioritized (while EBP referrals were not, for example) was that they were often tied to quality-based payment contracts. These complicated relationships formed the major priorities of the healthcare system and, thus, they helped to shape the jobs of clinicians.

Related to this, Not My Job emerged as a distinct theme to represent primary care clinicians' apparent lack of interest in carrying out the acts of referring patients to the CDSMP and/or other community resources. Indeed, primary care clinicians often reported that nurses and care coordinators had more time and more expertise when it came to discussing these sorts of opportunities with patients. As one physician said, "I am [only] trained in diagnosis and treatment." Because, in the minds of most clinicians, the CDSMP was not thought of as diagnosis or treatment, it could have been hard for clinicians to conceptualize the program as part of what they do. Certainly, clinicians had no frame of reference for what actually happened in the CDSMP because it was not part of their education or training. Consequently, clinicians felt both a lack of responsibility for and capability in making referrals. As one physician said.

"And the truth is that there are times I tell you that, you know I'm, I'm missing even I'm not gonna say more critical things but-but things I measure on. So...(sigh) is there, is there an automatic way to do this?"

The idea of "automating" the referral process was often explicitly suggested as a way to save time. Implicitly, however, it seemed that clinicians proposed the idea as a polite way of deferring responsibility for and attention to the task. It also may have represented a lack of interest in integrating the program into treatment plans. Along these lines, clinicians were quick to request that any information about the community programs or feedback related to when a patient participated be brief. It was not clear, for example, that clinicians had considered or desired a scenario by which they would adapt their care plans based on the goals identified or the lifestyle or self-care changes made by patients through program participation.

A notable exception to this was clinician C113. As the medical director of a rural practice, she had had the opportunity to attend a meeting in which a CDSMP participant had talked about her experience in the program. The clinician then familiarized herself with the program's curriculum and began referring some of her patients to the program. She had come to find so much value in the program, she was considering attending herself:

It would be kind of fun, actually, just to wouldn't it cuz I read the book, actually but just to sit in the class because, you know, I don't have half these issues, but there still are things in they're just lessons in life to learn, like, how to be happier and, you know, other things. So like I said, I have two [patients] that have gone through it and both of them have said that this is this has been a great program. So, again, I'm all in and if there's other ways, like I said, that you think that we can get this information out, I'm, I'm more than willing to, you know, try to do that.

In many ways, the enthusiasm and certainty with which C113 described the program's value was similar to that of many community stakeholders. What all these individuals had in common-and what distinguished them from most clinicians-was first-hand experience with the program itself and with its participants. The importance of this as a factor in increasing trust in and confidence about the program's value was consistent across clinician and stakeholder groups inside and 
outside the healthcare system. Indeed, it was those closest to the program that were most able to articulate how it helped people, often citing powerful anecdotes of participant success. As one program leader (S010) said:

The one thing that sticks out is people [with chronic conditions] think they're alone out there. So a lot of people with tears in their eyes would say, "I thought I was the only one feeling like this." And they realize that they're not. That everybody feels all of these things...no matter if it's diabetes, depression whatever it might be, they're all feeling the same thing. And the dynamics of the group interaction is, is the meeting. We're there as leaders but we're really just a guide. And they pretty much help each other. And they interact they hold each other accountable. We had one gentleman and wife who came. They came for him; he wasn't doing his homework. The other people in the class kept him accountable. And said, "Hey, you need to do this; your wife is emotional about this." And his wife was really the one who benefited the most from it as a care taker. And so when she left the last session she came up to me and she was crying. And she said, "Thank you for saving our marriage." So it so it can have really profound effects.

These effects were difficult for clinicians to fully appreciate, even after familiarizing themselves with evidence summaries of the program's value. "Seeing is Believing," captured this idea and represented the fact that clinicians who had no first-hand exposure to the CDSMP were forced to develop an impression through use of their imaginations. These impressions seemed to be reinforced by experiences with or impressions of other programs or interventions that patients pursued outside of the healthcare system. This connection raised concern in the minds of some that clinicians-especially "old fogeys"-would think of the CDSMP as "wishy washy" or alternative to effective care. Indeed, one clinician perceived the program as something that would be best suited for patients that were noncompliant with clinical care:

"So for instance somebody who doesn't necessarily trust medications or even though they may really need medications based on my recommendations wanna avoid meds, avoid drugs, find a more holistic approach to it. And I find that my knowledge there is fairly limited. So the yoga, the tai chi, and what not...".

In some ways, it seemed that the healthcare system was well positioned to reinforce a skepticism among clinicians about the value of all things outside its walls. Although this could be perceived as a reasonable and protective heuristic in many cases, it might have also predisposed clinicians to distrust EBPs and, potentially, focus less on the work that patients had do to self-manage their health. A key priority of the quantitative component was to further explore the validity of these ideas.

\section{Quantitative component Demographics}

In regards to that effort, we were successful in surveying 190 primary care clinicians from 4 of the 5 healthcare provider systems (and 9 of the 11 counties) in Southeast Minnesota. We did not receive responses to requests to contact clinicians from the smallest system, although there were few clinicians there, representing less than $5 \%$ of clinicians in the region. Approximately half (52\%) of the clinicians practiced in an urban/suburban community, while the rest were located in more rural settings. The response rate for the largest of the health systems was $37 \%(174 / 474)$. Response rates for the other systems were not calculable but were believed to range from 5 to $50 \%$ (the surveys were handed out in person to attendees of institutional healthcare provider meetings and exact denominators are not known). Through convenience and snowball sampling procedures, we distributed surveys to 167 non-clinician stakeholders. Of these, 88 (53\%) completed the survey, with respondents representing every county in SE MN. Table 3 describes the survey participants.

\section{PRECEDE analysis}

We selected 8 survey items to represent four of the predetermined PRECEDE constructs (we were not confident that any of the items corresponded to relevant policy issues). Broadly, the questions asked both clinicians and stakeholders about their perceptions of barriers to clinical referrals to the CDSMP and other community resources (see Table 4). Overall, respondents $(260,96 \%)$ overwhelmingly agreed that community resources were integral to effective primary care. They also highlighted lack of clinician education and awareness about the CDSMP (203, 73\%) and practical challenges in referring to community resources $(220,79 \%)$ as key barriers. Importantly, both clinicians and stakeholders (63 and 74\%, respectively) felt that community resources were underutilized and underemphasized in their organizations. However, clinicians were twice as likely to emphasize the need for community resources to be trustworthy and reliable than their stakeholder counterparts ( $67 \%$ vs $34 \%$, respectively; $\mathrm{p}=<$ 0.001). Additional details about the PRECEDE survey results, organized by construct and participant group are presented in Table 4.

\section{Emergent themes analysis}

To explore the validity and nuance of the theme of Two Systems Two Worlds, we selected out the stakeholders who worked within the healthcare system and combined them with the clinicians to create a "healthcare system group" of 223 participants. We then used the crossgroup comparisons for all PRECEDE factors between this group and the community system group of 55 
Table 3 Characteristics of survey study participants

\begin{tabular}{ll}
\hline Participant Characteristic & $\mathrm{N}(\%)$ \\
\hline Primary Care Clinicians N=190 & \\
Type of Practice & $101(53.2 \%)$ \\
Family Practice & $50(26.3 \%)$ \\
Internal Medicine & $27(14.2 \%)$ \\
Other (urgent care, ED) & \\
Degree & $107(56.3 \%)$ \\
MD/DO & $11(5.8 \%)$ \\
PA & $49(25.8 \%)$ \\
NP & $9(4.7 \%)$ \\
Other & \\
Other Stakeholders $N=88$ & \\
Role/interest in improving health in SEMN & $19(22 \%)$ \\
Health care employee that may refer patients & $12(14 \%)$ \\
to community resources & \\
Health care employee not likely to refer patients & $14(16 \%)$ \\
to community resources & $16(18 \%)$ \\
Public health & $8(9 \%)$ \\
Community-based agency or non-profit & $7(8 \%)$ \\
Patient & $1(1 \%)$ \\
Researcher & $1(1 \%)$ \\
Payer/Insurer & $1(1 \%)$ \\
Funder/Philanthropist & $2(2 \%)$ \\
Voluntractor & $7(8 \%)$ \\
\hline
\end{tabular}

"Missing responses not counted in percentages participants (e.g. the stakeholders that did not work within the healthcare system). This analysis did not change impressions greatly, but did reinforce key differences between community and healthcare-based stakeholders. Specifically, healthcare system stakeholders were significantly less aware $(p<0.001)$, less trusting $(p=0.02)$, and less concerned about the accessibility of $(\mathrm{p}<0.001)$ community resources than their communitybased counterparts.

We identified 3 survey measures that corresponded to the theme of Not My Job and analyzed these in the same way as the PRECEDE factors. Specifically, the items explored the extent to which clinicians desired or were willing to take on the work required for clinical integration of the CDSMP. Consistent with the theme, only a minority of clinicians $(62,34 \%)$ expressed concern about the lack of a system for making referrals and few (26, $14 \%)$ expressed a clear desire to receive feedback if and when their patients participated in the program. Not surprisingly, the vast majority $(151,84 \%)$ of clinicians reported that any system pursued to initiate referrals must fit into the electronic workflow. Non-clinician stakeholders expressed significantly less extreme perceptions of the Not My Job measures in all 3 cases.

Finally, we used the clinician distributions of all survey items representing the Predisposing factors as well as an item assessing clinicians' perceptions of whether community resources were underutilized in their practice across levels of exposure (some vs none) to the CDSMP to represent Seeing is Believing. We found that clinicians with exposure to the CDSMP were significantly less likely to cite education and awareness as a barrier to

Table 4 Dichotomized responses to PRECEDE survey items

\begin{tabular}{|c|c|c|c|c|}
\hline Survey Measure & Total $(n=278)$ & Clinicians $(n=190)$ & Stakeholders $(n=88)$ & $P$ value \\
\hline \multicolumn{5}{|l|}{ Predisposing Factors } \\
\hline $\begin{array}{l}\text { Believe community resources are important } \\
\text { parts of effective primary care }\end{array}$ & $260(95.9 \%)$ & $179(95.2 \%)$ & $81(97.6 \%)$ & 0.36 \\
\hline $\begin{array}{l}\text { Believe community resources need to be } \\
\text { reliable and trustworthy }\end{array}$ & $153(56.7 \%)$ & $125(66.8 \%)$ & $28(33.7 \%)$ & $<0.01$ \\
\hline $\begin{array}{l}\text { Believe lack of education and awareness } \\
\text { about program is barrier to CDSMP referral }{ }^{c}\end{array}$ & $203(73.0 \%)$ & $134(73.2 \%)$ & $69(86.3 \%)$ & 0.02 \\
\hline \multicolumn{5}{|l|}{ Reinforcing Factors } \\
\hline $\begin{array}{l}\text { Believe community resources, if suggested, } \\
\text { are not likely to be used by patients }{ }^{2}\end{array}$ & $43(15.9 \%)$ & $33(17.6 \%)$ & $10(12.0 \%)$ & 0.25 \\
\hline Believe community resources are not accessible ${ }^{b}$ & $193(71.5 \%)$ & $111(59.4 \%)$ & $82(98.8 \%)$ & $<0.01$ \\
\hline \multicolumn{5}{|l|}{ Enabling Factors } \\
\hline $\begin{array}{l}\text { Believe easy to make referrals to community } \\
\text { resources if desired }\end{array}$ & $43(15.9 \%)$ & $32(17.0 \%)$ & $11(13.3 \%)$ & 0.43 \\
\hline \multicolumn{5}{|l|}{ Administrative Issues } \\
\hline $\begin{array}{l}\text { Believe community resources are emphasized } \\
\text { and encouraged in my setting }\end{array}$ & $114(42.2 \%)$ & $76(40.4 \%)$ & $38(46.3 \%)$ & 0.37 \\
\hline $\begin{array}{l}\text { Believe community resources are underutilized } \\
\text { in my setting }\end{array}$ & $180(66.7 \%)$ & $119(63.3 \%)$ & $61(74.4 \%)$ & 0.08 \\
\hline
\end{tabular}

${ }^{\mathrm{a}}$ Missing 7 responses ( 2 clinicians); ${ }^{\mathrm{b}}$ Missing 8 responses ( 3 clinicians); ${ }^{\mathrm{c}}$ Missing 15 responses ( 7 clinicians); ${ }^{\mathrm{d}}$ Missing 8 responses ( 2 clinicians) 
referral $(p<0.0001)$. Exposure to the CDSMP was not significantly associated with better perceptions of community resources in general, however. This supports the idea that the CDSMP and other EBPs may not be viewed in the same light as other, more typical and "trustworthy" community resources. Other details about the quantitative analyses of the emergent themes are presented in Table 5.

\section{Discussion}

\section{Our findings}

We sought to understand, categorize, and richly describe key challenges and opportunities related to integrating EBPs into routine primary care practice in the United States as part of a parent, participatory action research project. Three themes emerged from this investigation that help explain why primary care and communitybased programs exist in disconnected worlds.

\section{Limitations and strengths}

Our study has several weaknesses and, partly for this reason, it should be viewed as exploratory. Small numbers of interview participants among some stakeholder constituencies, although representative of Southeast Minnesota, could have prevented us from reaching a generalizable saturation of insights. Sampling bias, due in part to sampling procedures and low response rates, may threaten the validity of the survey data. Additionally, our surveys were developed to explore topics consistent with PRECEDE constructs and to triangulate with qualitative data. They were not designed to measure PRECEDE constructs themselves, nor was any reliability or validity testing done. Although not feasible for our study, the survey likely would have been more useful had it been designed after completion of the qualitative component. Our focus on a large, 11-county region of Southeast Minnesota, albeit relevant to our purpose, may also threaten the applicability of our findings. The validity of our findings is strengthened, however, by the sampling of an entire geographic region, our conduct of quantitative analyses after and in confirmation of qualitative data, and the congruence of findings across methods and participants. Our study also adds to the body of literature related to EBPs in that-while many studies focused on EBP effectiveness, acceptability, and value, have been conducted [34-37] - to our knowledge, this is the first empirical, theory-guided exploration of the feasibility of clinical integration of community-based EBPs. In that respect, this study provides timely evidence to stakeholders seeking to scale EBPs and improve population health.

\section{Conclusions}

Our findings suggest that key policy and administrative issues have played important roles in prescribing-in a very fragmented and siloed way-the work that healthcare and the community are "supposed to do" to contribute to health. Two separate systems with distinct cultural identities coexist and do not understand each other well. Our work supports prior laments describing healthcare as a "sick care" system [38], for example, but adds deeper understanding of the pathways by which healthcare is directed to focus somewhat exclusively on the activities of "diagnosis and treatment." It also begins to articulate the role EBPs may play in person-centered and evidence-based efforts to improve population health. Indeed, to the extent "well-care" is needed and can be operationalized through the delivery of EBPs, the community appears capable and motivated to contribute. Its capacity to do this at scale and in a way healthcare will understand and accept-and eventually embrace, coordinate with, and seamlessly integrate-is likely to require bidirectional education and engagement, as well as policy and system-level changes (see Fig. 2).

\section{Practice implications}

Stakeholders guiding efforts to transform healthcare delivery in the United States should consider our findings. Specifically, the Department of Health and Human Services has made CDSMP implementation a key goal in its framework for improving the health of patients with multiple chronic conditions [39], and the $\mathrm{CDC}$ and the Centers for Medicare and Medicaid Services (CMS) have rolled out initiatives to implement and pay for the Diabetes Prevention Program, respectively. For the most part, these and other approaches to EBP implementation have relied on community-based and publicly-funded dissemination channels, and only assumed the participation of primary care. Our findings suggest that this strategy is unlikely to penetrate the healthcare system or influence its priorities. Indeed, to the extent disease experts-who may be trained only in the activities of "diagnosis and treatment"-continue to develop and inform the "metrics and measures" that determine what primary care clinicians know, do, and get paid for [40, 41], actual referrals to EBPs and incorporation of them into treatment plans are unlikely to come at scale.

\section{Future directions}

For this and other reasons, some might argue that the potential benefits of integrating EBPs into clinical care are not worth the effort and resources. We are wary of this logic and warn of the potentially negative consequences of community-based implementation 
Table 5 Emergent themes analyses

\begin{tabular}{|c|c|c|c|c|}
\hline \multicolumn{5}{|l|}{ Two Systems Two Worlds } \\
\hline Survey Item & Total $(n=278)$ & Healthcare system $(n=223)$ & Community system $(n=55)$ & $P$ value \\
\hline \multicolumn{5}{|l|}{ Predisposing Factors } \\
\hline $\begin{array}{l}\text { Believe community resources are } \\
\text { important parts of effective primary care }{ }^{a}\end{array}$ & $260(93.5 \%)$ & $209(93.7 \%)$ & $51(92.7 \%)$ & 0.39 \\
\hline $\begin{array}{l}\text { Believe community resources need to be } \\
\text { reliable and trustworthy }{ }^{b}\end{array}$ & $153(55.0 \%)$ & $131(58.7 \%)$ & $22(40.0 \%)$ & 0.02 \\
\hline $\begin{array}{l}\text { Believe community resources are something } \\
\text { I am aware of and educated about }{ }^{\mathrm{b}}\end{array}$ & $78(28.1 \%)$ & $74(33.2 \%)$ & $4(7.3 \%)$ & $<0.01$ \\
\hline \multicolumn{5}{|l|}{ Reinforcing Factors } \\
\hline $\begin{array}{l}\text { Believe community resources, if suggested, } \\
\text { are not likely to be used by patients } s^{\mathrm{a}}\end{array}$ & $43(15.5 \%)$ & $39(17.5 \%)$ & $4(7.3 \%)$ & 0.07 \\
\hline $\begin{array}{l}\text { Believe community resources are not } \\
\text { accessible }^{b}\end{array}$ & $193(69.4 \%)$ & $142(63.7 \%)$ & $51(92.7 \%)$ & $<0.01$ \\
\hline \multicolumn{5}{|l|}{ Enabling Factors } \\
\hline $\begin{array}{l}\text { Believe easy to make referrals to } \\
\text { community resources if desired }^{a}\end{array}$ & $43(15.5 \%)$ & $36(16.1 \%)$ & $7(12.7 \%)$ & 0.60 \\
\hline \multicolumn{5}{|l|}{ Administrative Issues } \\
\hline $\begin{array}{l}\text { Believe community resources are } \\
\text { emphasized and encouraged in my }^{\text {setting }}{ }^{c}\end{array}$ & $114(41.0 \%)$ & 89 (39.9\%) & $25(45.5 \%)$ & 0.28 \\
\hline $\begin{array}{l}\text { Believe community resources are } \\
\text { underutilized in my setting }\end{array}$ & $180(64.7 \%)$ & $146(65.5 \%)$ & $34(61.8 \%)$ & 1.00 \\
\hline \multicolumn{5}{|l|}{ Not My Job } \\
\hline Survey Item & Total $(n=278)$ & Clinicians ( $n=190)$ & Stakeholders $(n=88)$ & $P$ Value \\
\hline $\begin{array}{l}\text { Believe lack of feedback about } \\
\text { patient participation is barrier } \\
\text { to referral }^{d}\end{array}$ & $62(22.3 \%)$ & $26(14.2 \%)$ & $36(45.0 \%)$ & $<0.01$ \\
\hline $\begin{array}{l}\text { Believe lack of an electronic referral } \\
\text { system is barrier to referral }^{d}\end{array}$ & $105(37.8 \%)$ & $62(33.9 \%)$ & $43(53.8 \%)$ & $<0.01$ \\
\hline $\begin{array}{l}\text { Believe referral process, if pursued, } \\
\text { must be integrated into work flow }\end{array}$ & 209 (75.2\%) & $151(84.4 \%)$ & $58(69.9 \%)$ & $<0.01$ \\
\hline \multicolumn{5}{|l|}{ Seeing is Believing } \\
\hline Survey Item & $\begin{array}{l}\text { All clinicians } \\
\left(n=186^{6}\right)\end{array}$ & $\begin{array}{l}\text { Clinicians with CDSMP exposure } \\
(n=38)\end{array}$ & $\begin{array}{l}\text { Clinicians w/out CDSMP exposure } \\
(n=148)\end{array}$ & $P$ value \\
\hline $\begin{array}{l}\text { Believe community resources are } \\
\text { important part of primary care }\end{array}$ & $177(95.2 \%)$ & $35(92.1 \%)$ & $142(96.0 \%)$ & 0.33 \\
\hline $\begin{array}{l}\text { Believe community resources need } \\
\text { to be reliable and trustworthy }{ }^{g}\end{array}$ & $123(66.5 \%)$ & $23(62.2 \%)$ & $100(67.6 \%)$ & 0.53 \\
\hline $\begin{array}{l}\text { Believe education and awareness } \\
\text { about CDSMP is barrier to referral }\left.\right|^{h}\end{array}$ & $134(73.2 \%)$ & 7 (18.9\%) & 127 (87.0\%) & $<.0001$ \\
\hline $\begin{array}{l}\text { Believe community resources are } \\
\text { underutilized in their setting }\end{array}$ & 118 (63.4\%) & $23(60.5 \%)$ & 95 (64.2\%) & 0.68 \\
\hline
\end{tabular}

that is entirely independent of healthcare. For example, we uncovered numerous examples of clinician distrust of EBPs and concerns about the extent to which these programs are perceived to "compete with" clinical care. If explicit efforts are not undertaken to engage clinicians-potentially through experiential learning and detailing-we foresee a scenario by which clinicians become less supportive of and potentially opposed to EBPs. This would undermine the community's efforts. We believe future work should focus on the development and testing of multi-level implementation strategies that seek to engage clinicians and broaden the priorities of healthcare. Such strategies should be aligned to counteract the barriers 
identified here (see Fig. 2) [42]. They should promote and reward the establishment of clinic-community partnerships and align with system and reimbursement model transformations presently occurring in the United States [43]. Specifically, the value of reorganizing, reframing, and repurposing the community and articulating its potential and non-duplicative role as a "well-care" system of EBPs should be explored. We believe this could facilitate the creation and orient the function of scalable clinic-community partnerships that improve population health. Indeed, this is the approach our own community-partnered team has taken as part of the parent project. Thus far, it has spurred the creation of a "hub-based" [44] model for EBP implementation and an accompanying technology (www.wellconnectsemn.org). We are engaging with learning collaboratives convened by the National Council on Aging and others interested in similar approaches to explore opportunities for refinement and evaluation.

\section{Additional files}

Additional file 1: a) Primary Care Clinician Survey. b) Stakeholder Survey. c) Participant Interview Guide. d) Sample Participant Quotes by PRECEDE Construct (PDF $784 \mathrm{~kb}$ )

\section{Abbreviations}

AHRQ: The Agency for Healthcare Research and Quality; CDC: The U.S. Centers for Disease Control and Prevention; CDSMP: The Chronic Disease Self-Management Program; CMS: The U.S. Centers for Medicare and Medicaid Services; DHHS: The U.S. Department of Health and Human Services; EBPs: Evidence-based health promotion programs; PRECEDE: Predisposing, Reinforcing, and Enabling Constructs in Diagnosis and Evaluation and the associated implementation planning model

\section{Acknowledgements}

Not applicable.

\section{Funding}

This publication was made possible by CTSA Grant Number UL1 TR000135 from the National Center for Advancing Translational Sciences (NCATS), a component of the National Institutes of Health (NIH). Its contents are solely the responsibility of the authors and do not necessarily represent the official view of $\mathrm{NIH}$.

\section{Availability of data and materials}

Much of the data generated or analyzed during this study are included in this published article and its Additional file 1. All additional datasets and analyses are available from the corresponding author on reasonable request.

\footnotetext{
Authors' contributions

ALL was the principal investigator of the study. He conceptualized the overall study design and oversaw all aspects of stakeholder engagement: participant recruitment; and data collection, analysis, and reporting. He wrote the first draft of the manuscript, oversaw the editing and revision process, and approved the final draft. KS led in the qualitative analysis and in the identification of emergent themes and contributed to several drafts of the manuscript. JE conducted all clinician interviews, coded all interviews, and contributed to the development of the qualitative framework analyses and to the writing of the final manuscript. SD assisted in the recruitment of participants, coded all interviews, and contributed to the identification of qualitative themes and to the writing of the final manuscript. MB conducted all quantitative and statistical analyses, reviewed all drafts of the manuscript,
}

and contributed to the writing of the final version. LC conducted clinician interviews, reviewed multiple drafts of the manuscript, and contributed to the writing of the final version. NB conducted clinician interviews, reviewed multiple drafts of the manuscript, and contributed to the writing of the final version. CG assisted with quantitative analyses and with the creation of tables, reviewed multiple drafts of the manuscript, and contributed to the final version. VM provided significant feedback and editing to late versions of the manuscript. All authors read and approved the final manuscript.

\section{Authors' information}

ALL is a physician and implementation scientist in the Knowledge and Evaluation Research Unit at Mayo Clinic.

\section{Ethics approval and consent to participate}

This study was approved by the Mayo Clinic Institutional Review Board through its Office of Community Engagement in Research under exempt status. All participants gave consent to participate in this study. The reference number for the study is: 15-002587-03

\section{Consent for publication}

Not applicable.

\section{Competing interests}

The authors declare that they have no competing interests.

\section{Publisher's Note}

Springer Nature remains neutral with regard to jurisdictional claims in published maps and institutional affiliations.

\section{Author details}

${ }^{1}$ Knowledge and Evaluation Research Unit, Mayo Clinic, 200 First Street SW, Rochester, MN 55905, USA. ²Division of Health Care and Policy Research, Mayo Clinic, 200 First Street SW, Rochester, MN 55905, USA. ${ }^{3}$ Southeastern Minnesota Area Agency on Aging, 2720 Superior Drive NW, Suite 102, Rochester, MN 55901, USA. ${ }^{4}$ Dan Abraham Healthy Living Center, Mayo Clinic, 200 First Street SW, Rochester, MN 55905, USA. ${ }^{5}$ Mayo Clinic School of Medicine, Mayo Clinic, 200 First Street SW, Rochester, MN 55905, USA.

Received: 30 January 2017 Accepted: 21 January 2018

Published online: 31 January 2018

\section{References}

1. Ward BW, Schiller JS, Goodman RA. Multiple chronic conditions among US adults: a 2012 update. Prev Chronic Dis. 2014;11:E62.

2. Bodenheimer $\mathrm{T}$, Lorig $\mathrm{K}$, Holman $\mathrm{H}$, Grumbach $\mathrm{K}$. Patient self-management of chronic disease in primary care. JAMA. 2002;288(19):2469-75.

3. Norris SL, Engelgau MM, Narayan KV. Effectiveness of self-management training in type 2 diabetes a systematic review of randomized controlled trials. Diabetes Care. 2001;24(3):561-87.

4. Gallant MP. The influence of social support on chronic illness selfmanagement: a review and directions for research. Health Educ Behav. 2003;30(2):170-95.

5. Cunningham PJ. Chronic burdens: the persistently high out-of-pocket health care expenses faced by many Americans with chronic conditions Issue Brief (Commonw Fund). 2009;63:1-14.

6. Gallacher K, May CR, Montori VM, Mair FS. Understanding patients' experiences of treatment burden in chronic heart failure using normalization process theory. Ann Fam Med. 2011;9(3):235-43.

7. Wagner EH, Austin BT, Davis C, Hindmarsh M, Schaefer J, Bonomi A. Improving chronic illness care: translating evidence into action. Health Aff (Millwood). 2001;20(6):64-78.

8. Sequist TD, Taveras EM. Clinic-community linkages for high-value care. N Engl J Med. 2014;371(23):2148-50.

9. Herrin J, St Andre J, Kenward K, Joshi MS, Audet AM, Hines SC. Community factors and hospital readmission rates. Health Serv Res. 2015;50(1):20-39.

10. Parekh AK, Kronick R, Tavenner M. Optimizing health for persons with multiple chronic conditions. JAMA. 2014;312(12):1199-200.

11. Ory MG, Smith ML, Patton K, Lorig K, Zenker W, Whitelaw N. Selfmanagement at the tipping point: reaching 100,000 Americans with evidence-based programs. J Am Geriatr Soc. 2013;61(5):821-3. 
12. US Department of Health and Human Services: Multiple chronic conditions - a strategic framework: optimum health and quality of life for individuals with multiple chronic conditions. Washington, DC, Department of Health and Human Services 2010.

13. Lorig KR, Sobel DS, Ritter PL, Laurent D, Hobbs M. Effect of a selfmanagement program on patients with chronic disease. Eff Clin Pract. 2001;4(6):256-62.

14. Ory $M G$, Ahn $S$, Jiang $L$, Smith $M L$, Ritter $P L$, Whitelaw $N$, Lorig K. Successes of a national study of the chronic disease self-management program: meeting the triple aim of health care reform. Med Care. 2013:51(11):992-8.

15. U.S. Department of Health \& Human Services: Independent experts confirm that diabetes prevention model supported by the Affordable Care Act saves money and improves health. In. http://www.hhs.gov/about/news/2016/03/ 23/independent-experts-confirm-diabetes-prevention-model-supportedaffordable-care-act-saves-money.html; 2016.

16. Samuel-Hodge CD, Keyserling TC, Park S, Johnston LF, Gizlice Z, Bangdiwala SI. A randomized trial of a church-based diabetes self-management program for African Americans with type 2 diabetes. Diabetes Educ. 2009; 35(3):439-54.

17. Lorig K, Ritter PL, Villa FJ, Armas J. Community-based peer-led diabetes selfmanagement: a randomized trial. Diabetes Educ. 2009;35(4):641-51.

18. Lorig K, Ritter PL, Villa F, Piette JD. Spanish diabetes self-management with and without automated telephone reinforcement: two randomized trials. Diabetes Care. 2008;31(3):408-14.

19. Li F, Harmer P, Fisher KJ, McAuley E, Chaumeton N, Eckstrom E, Wilson NL. Tai chi and fall reductions in older adults: a randomized controlled trial. J Gerontol A Biol Sci Med Sci. 2005;60(2):187-94.

20. Clemson L, Cumming RG, Kendig H, Swann M, Heard R, Taylor K. The effectiveness of a community-based program for reducing the incidence of falls in the elderly: a randomized trial. J Am Geriatr Soc. 2004;52(9):1487-94.

21. Brady TJ ML, Beauchesne D, Bhalakla A, Chervin D, Daniels B, Greenberg M, House M, O'Coleman B: Sorting through the evidence for the Arthritis SelfManagement Program and the Chronic Disease Self-Managment Program, Executive Summary of ASMP/CDSMP meta-analyses. In.; May 2011.

22. Brady TJ, Sniezek J, Ramsey LA. News from the CDC: scaling up sustainable intervention delivery-lessons learned from the CDC arthritis program. Transl Behav Med. 2012;2(1):3-5.

23. Shier V, Trieu E, Ganz DA. Implementing exercise programs to prevent falls: systematic descriptive review. Inj Epidemiol. 2016;3:16.

24. Green L KM: Health promotion planning: an educational and ecological approach, 4th edition edn. Mountain View, CA: Mayfield Publishers; 2005

25. Crosby R, Noar SM. What is a planning model? An introduction to PRECEDEPROCEED. J Public Health Dent. 2011;71(Suppl 1):S7-15.

26. Whyte WF. (Ed.). Sage focus editions, Vol. 123. Participatory action research. Thousand Oaks: Sage Publications; 1991.

27. Jagosh J, Macaulay AC, Pluye P, Salsberg J, Bush PL, Henderson J, Sirett E, Wong G, Cargo M, Herbert CP. Uncovering the benefits of participatory research: implications of a realist review for health research and practice. Milt Q. 2012:90(2):311-46.

28. Minkler M. Using participatory action research to build healthy communities. Public Health Rep. 2000;115(2-3):191.

29. Published Applications of the Precede Model. http://www.lgreen.net/ precede.htm.

30. Creswell JW, Klassen AC, Plano Clark VL, Smith KC. Best practices for mixed methods research in the health sciences. National Institutes of Health: Bethesda (Maryland); 2011.

31. Green CA, Duan N, Gibbons RD, Hoagwood KE, Palinkas LA, Wisdom JP. Approaches to mixed methods dissemination and implementation research: methods, strengths, caveats, and opportunities. Admin Pol Ment Health. 2015;42(5):508-23.

32. Galesic M, Bosnjak M. Effects of questionnaire length on participation and indicators of response quality in a web survey. Public Opin. Q. 2009;73(2):349-60

33. Harris PA, Taylor R, Thielke R, Payne J, Gonzalez N, Conde JG. Research electronic data capture (REDCap)-a metadata-driven methodology and workflow process for providing translational research informatics support. J Biomed Inform. 2009;42(2):377-81.
34. Palmer RC, Batra A, Anderson C, Page T, Vieira E, Seff L. Implementation of an evidence-based exercise program for older adults in South Florida. J Aging Res. 2016;2016:9630241.

35. Tomioka M, Braun KL. Implementing evidence-based programs: a fourstep protocol for assuring replication with fidelity. Health Promot Pract. 2013;14(6):850-8.

36. Snyder SJ, Thompson M, Denison P: EnhanceFitness: a 20-year dissemination history. Front Public Health. 2014;2:270.

37. Glasgow RE, Vogt TM, Boles SM. Evaluating the public health impact of health promotion interventions: the RE-AIM framework. Am J Public Health. 1999:89(9):1322-7.

38. Fani Marvasti F, Stafford RS. From sick care to health care-reengineering prevention into the U.S. system. N Engl J Med. 2012;367(10):889-91.

39. United States Department of Health and Human Services (2014). "HHS Initiative on Multiple Chronic Conditions." Retrieved January 20, 2014, from http://www.hhs.gov/ash/initiatives/mcc/.

40. Kane RL. Creating practice guidelines: the dangers of over-reliance on expert judgment. J Law Med Ethics. 1995;23(1):62-4.

41. Walter LC, Davidowitz NP, Heineken PA, Covinsky KE. Pitfalls of converting practice guidelines into quality measures: lessons learned from a VA performance measure. JAMA. 2004;291(20):2466-70.

42. Grol R, Grimshaw J. Evidence-based implementation of evidence-based medicine. Jt Comm J Qual Improv. 1999;25(10):503-13.

43. Rajkumar R, Conway PH, Tavenner M. CMS-engaging multiple payers in payment reform. JAMA. 2014;311(19):1967-8.

44. Schwamm LH, Audebert HJ, Amarenco P, Chumbler NR, Frankel MR, George MG, Gorelick PB, Horton KB, Kaste M, Lackland DT, et al. Recommendations for the implementation of telemedicine within stroke systems of care: a policy statement from the American Heart Association. Stroke. 2009;40(7): 2635-60.

\section{Submit your next manuscript to BioMed Central and we will help you at every step:}

- We accept pre-submission inquiries

- Our selector tool helps you to find the most relevant journal

- We provide round the clock customer support

- Convenient online submission

- Thorough peer review

- Inclusion in PubMed and all major indexing services

- Maximum visibility for your research

Submit your manuscript at www.biomedcentral.com/submit 